\title{
Transmission Control Technology in Computer Wireless Network Communication
}

\author{
Feng Ling ${ }^{1,3^{*}}$, Rajamohan Parthasarathy ${ }^{2}$ \\ ${ }^{1}$ Faculty of Engineering, Built Environment and Information Technology, SEGi University, Petaling Jaya 47810, Malaysia \\ ${ }^{2} \mathrm{PhD}$ Research Supervisor, Faculty of Engineering, Built Environment and Information Technology, SEGi University, Petaling Jaya 47810, Malaysia \\ ${ }^{3}$ Faculty of Engineering, Lishui University, Lishui 323000, Zhejiang Province, China. E-mail: lintel_1@qq.com
}

\begin{abstract}
With the rapid development of wireless network communication in China, information transmission technology has become the key point. Information transmission mainly includes wireline network communication, wireless network communication and broadband communication. Due to the limitation of communication barriers in different areas and environment, problems, such as poor signal, weak channel transmission and distribution ability, and channel congestion, will emerge during the information transmission process. Therefore, by taking transmission control technology as the research object, analysis on the disadvantages of computer wireless network communication are conducted in this article. Some corresponding improvement measures are also put forward for reference.
\end{abstract}

Keywords: Network Communication Technology; Network Transmission Control; Breakout Chain; Data Delay

\section{Introduction}

By using network transmission control technology, computer wireless network communication realizes the rapid transmission of messages. With the combination of computer technology and communication technology, all kinds of messages can be transmitted through computer equipment. Therefore, relevant researchers have deeply analyses and studies on computer network communication technology. Meanwhile, complicated information and a large number of resources are effectively solved and quickly transmitted through transmission control technology, which improves the efficiency of automatic office work.

\section{The characteristics of computer network communication technology}

2.1 The meaning and composition of com-

\section{puter wireless network communication}

There are three aspects in the overall system of computer network communication. The first is software equipment; the second is hardware equipment; the third is communication protocol. Meanwhile, the system can be also divided into other three aspects according to the specific structure. The first is host; the second is communication subnet; the third is resource subnet. No matter what kind of structure "communication" is constituted, it is the key channel for its connection, and is the bridge for the transmission of various information and a large amount of data in the computer network communication system. Therefore, improving the part of wireless network communication of computers can effectively enhance the operation effect and work quality of the whole system to ensure the transmission speed and

\footnotetext{
Copyright (C) 2020 Feng Ling and Rajamohan Parthasarathy

doi: 10.18282/ii.v2i1.334

This is an open-access article distributed under the terms of the Creative Commons Attribution Non-Commercial License

(http://creativecommons.org/licenses/by-nc/4.0/), which permits unrestricted non-commercial use, distribution, and reproduction in any medium, provided the original work is properly cited.
} 
quality of information ${ }^{[1]}$.

\subsection{Innovative network communication technology}

In the environment of modern information society, computer technology has been popularized, which has promoted the development of China's society. Wireless network communication technology is a technology developed from the combination of network and computer technology. While upgrading basic technology, human beings are constantly improving and innovating the technology, making it better meet the needs and keep pace with the times.

\subsection{Diversified network communication technology}

Computer wireless network communication technology has been widely popularized as the deepening development of Internet technology. It is the latest communication mode. Wireless network communication can meet the basically transmission needs of all kinds of data and information, and there are many ways and means in the transmission process. Computer network technology is the most important support point. The advanced research results of unified communication science and technology make the network communication means show the development characteristics of modernization and diversity ${ }^{[2]}$.

\section{Problems of transmission control technology}

\subsection{Symbol error}

Wireless network communication can transmit data both outdoors and indoors. In the transmission process, transmissions are bound to be affected and interfered by various external objects, especially objects that are too large with very smooth surface. The signals in information transmission also have certain interference. When the interference is serious, it will cause the loss or destruction of data in the transmission process, which will seriously affect the information transmission quality of wireless network communication system. Normally, when the client mobile terminal requests information transmission from the host, a second time of transmission can be requested if the corresponding information packet is not received or the information is incomplete. The transmission terminal will carry out information transmission again, thus increasing the probability of congestion in the wireless network communication system, resulting in the loss of information in the information channel ${ }^{[3]}$.

\subsection{Data delay}

At present, the customer's mobile terminal will move constantly in the coverage area of the wireless network in the operation of the wireless network communication system, which will lead to the constantly changing of information transmission point. In this case, stricter requirements on the functions of transmission speed and transmission fixed point determination of the information transmission terminal is required. At present, the MANET network used in the system is easy to cause information loss in changing the information transmission channel, leading to plenty of transmission request applied by customers. Correspondingly, the information transmission terminal will send information many times, and thus causes congestion in the wireless network communication system and the date transmission time will be delayed. Therefore, it is very important to improve the network transmission control system.

\section{Improvements of transmission control technology in computer wireless network communication}

\subsection{Measures for breakout chain improve- ments}

At present, relevant researchers have been working on solving the problems of wireless network communication port and network timeout in the development process of computer wireless network communication technology. They seek to master the actual situation of transportation road disconnection, and control window string and transportation channel. The transmission port of data should adopt wireless network node. With the assistance of the node, the current server wireless network communication signal should be connected to find out whether the channel is interrupted, and then deal with the problems with the combination of the actual situation. At the same time, the window string is also an important warning signal to prompt the interruption of the current channel. It is necessary to freeze the information of the 
current serial port, suspend the delayed transmission of information, or re-transmit it over time. after the transmission port receives the signal. Then, according to the situation of channel interruption, the problem is re-detected, and the result is sent to the customers for data retransmission ${ }^{[4]}$.

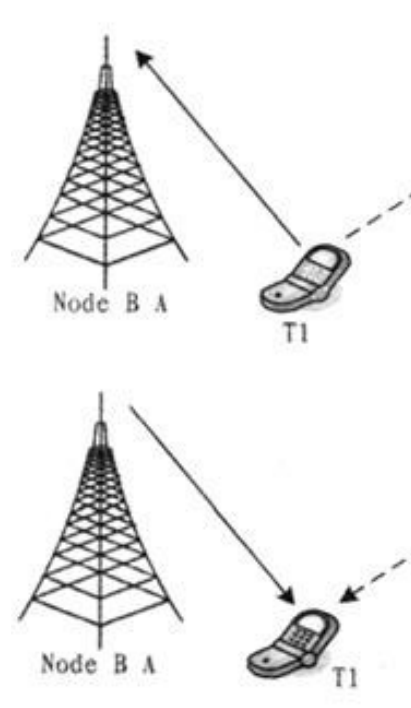

(a) uplink

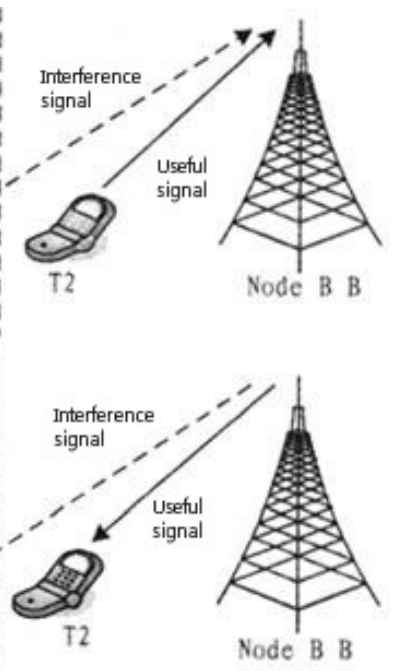

(b) down link

Figure 1. Link interruption in computer communication system.

\subsection{Wireless transmission control protocol}

With the help of eNodeB base station, wireless transmission control protocol (WTCP) transfer TCP data to minimize congestion caused by packet loss in wireless network. However, WTCP does not set a corresponding timeout timer to measure the time spent on data retransmission. Therefore, the round-trip delay of data transmission in WTCP will not be affected by the retransmission of data packets in eNodeB base station. Receiver will send an application to the data server in connecting network by using WTCP. Then, the eNodeB base station will send a corresponding string message to the user's terminal, indicating that the request from the user has been received. When the connection between the transmitter and the receiving end is established, data information transmission of different network terminals can be realized. The eNodeB base station includes network levels include WTCP and IP. WTCP can cache data packets in IP. For those data packets that do not meet the sequence requirements, WTCP will consider them as channel link errors, and control the end-to-end data transmission delay by setting the congestion window at the sender. The application of ITCP in wireless communication transmission control mainly increases the data throughput of the link, and reduces the bit error rate of network channel ${ }^{[5]}$. 


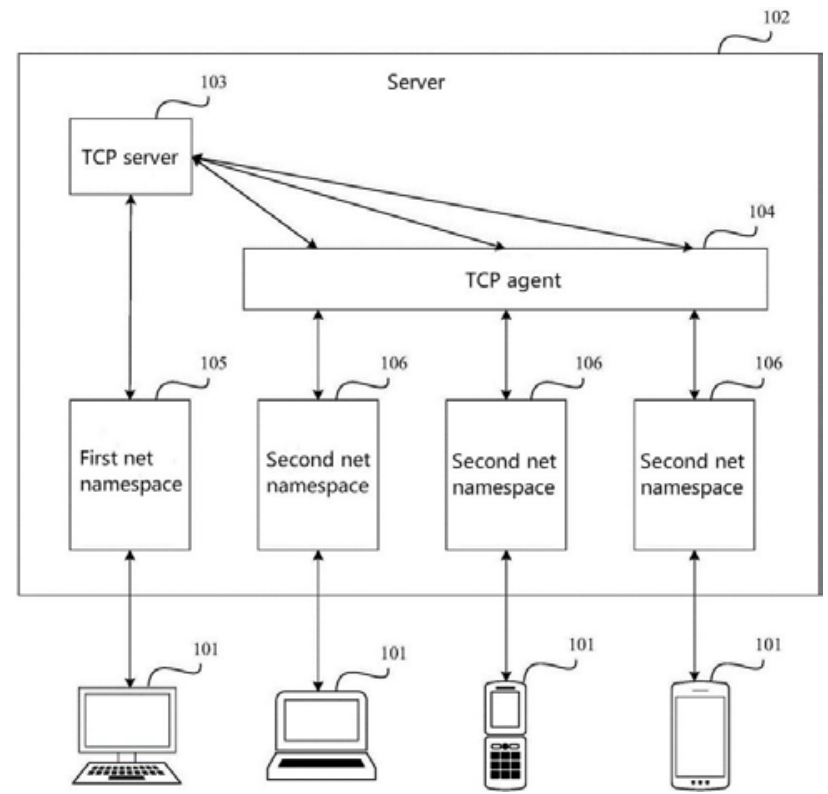

Figure 2. End-to-end communication control in wireless network communication.

\subsection{Transmission control of retransmission loss detection and discrimination in wireless communication}

Because of the high bit error rate of data transmission in wireless channel, data packet loss or retransmission can be effectively reduced by controlling congestion window and data retransmission through retransmission loss detection and discrimination agent. Retransmission loss detection and discrimination (DDLRP) usually uses congestion warning (CW) to detect channel congestion or non-congestion packet loss. In case of information transmission congestion at the transmitter, the router will send a string congestion warning to inform the sender to retransmit the data and detect the lost data packet. When the received congestion warning is 1 , the congestion control of lost data packets will be started. When the received warning is 0 , it indicates that the packet loss is not caused by congestion.

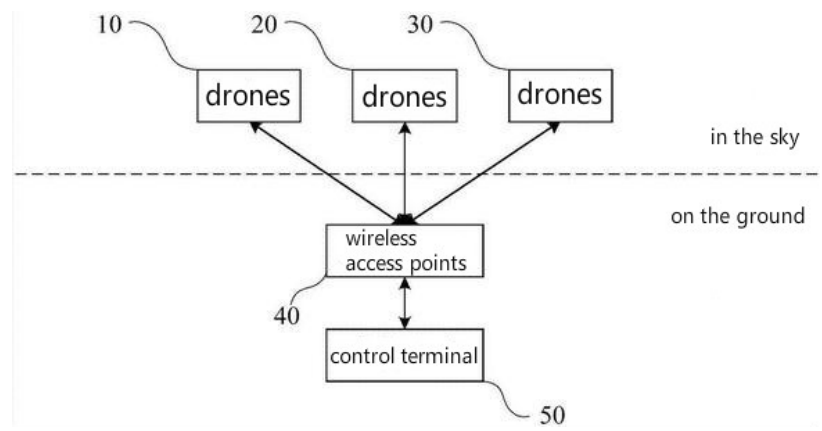

Figure 3. Loss detection of re-transmission and DDLRP transmission control.

\subsection{Measures for data delay improvements}

At present, the bandwidth of the sending end is larger than that of the receiving end in the network communication and data transmission. Moreover, the data delay variation in round-trip transmission process is caused by the use of wireless transmission bandwidth by multiple users, which leads to the reduction of data throughput in network transmission control. Through the solution to data delay variation of non-congestion loss control (TCP NCL), the problem in network links can be improved, thus reducing the overtime retransmission of lost data packets by the sender. By using congestion determination timer, timeout retransmission timer and so on, congestion can be controlled in the process of data retransmission. First of all, when the congestion determination timer starts, the string data received by the sender can be detected, and the main reasons for the timeout retransmission phenomenon can be analyzed. It is necessary to confirm the retransmitted data packet if time-out 
retransmission is caused by data packet loss; the data packet round-trip delay should be updated without enabling congestion control, if time-out retransmission is leaded by data delay variation, to ensure stable performance in wireless communication transmission.

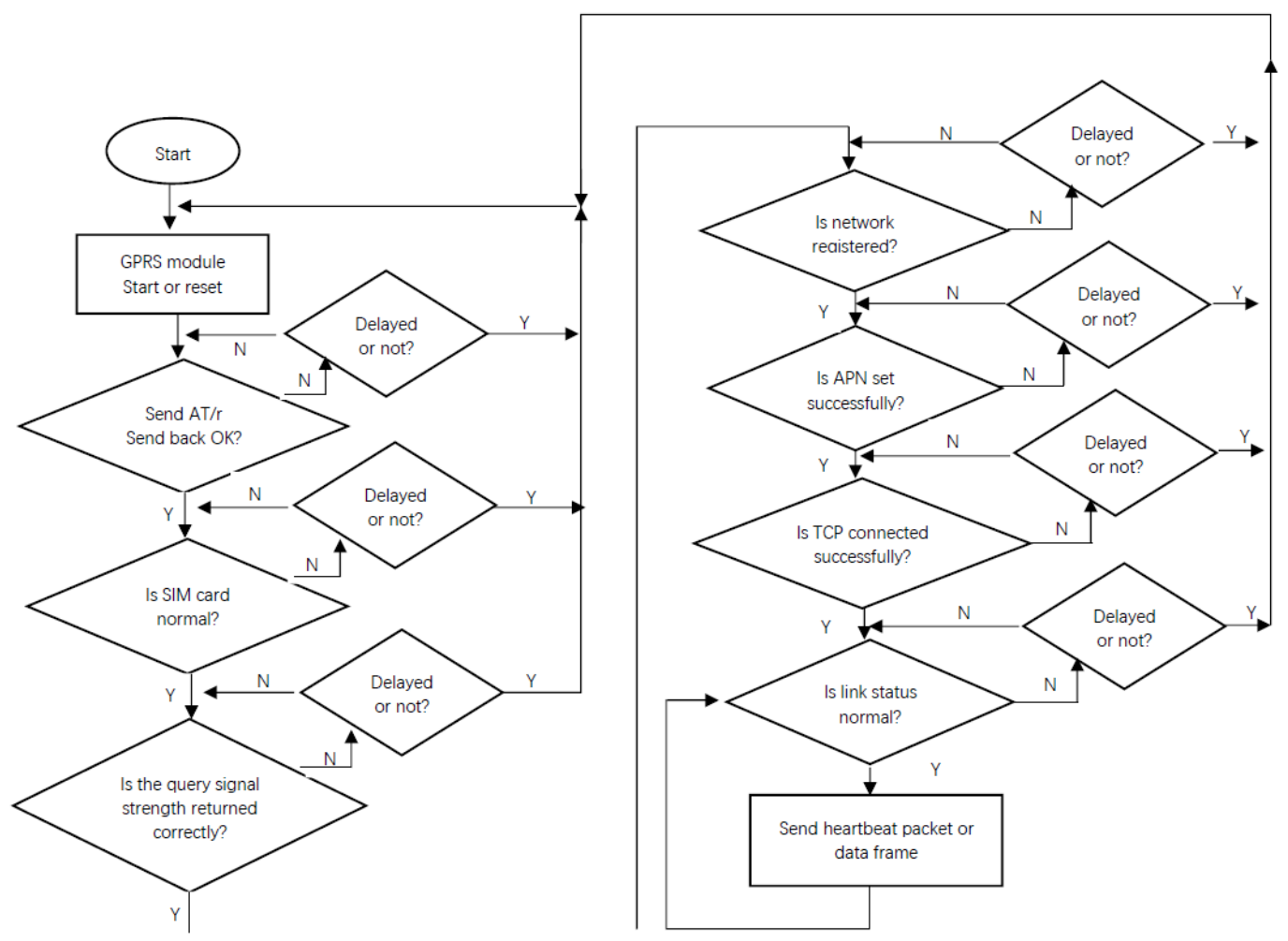

Figure 4. TCP NCL program.

\subsection{Improvement measures for snoop packet capture}

Snoop can also effectively calculate the number of information packets and lost packets in the transmission. For those unrecognizable data packets, snoop can cache them temporarily for further discrimination. According to the captured data packets, the agent labels and displays them with the help of computer language, and records the relevant communication protocols. In the wireless communication platform, it can play its role in the network transport layer, monitor the data packets and data resources in the base station, and prevent the data packets from losing or error codes. For those possible problems, snoop resends accurate data packets to control the channel bit error rate of network communication signals at ports in time in order to prevent the error problems from being transmitted to the next layer. Besides, snoop can also make statistics on the loss of data packets, and calculate their number of real-time transmissions. If unidentified data packets are found during the statistics, snoop will cache the data packets and further analyze them. The captured data packets will be marked with computer language. The data packets can be recorded accurately in the information transport layer based on the marked display content.

\section{Conclusion}

In summary, information processing and transmission has been paid attention to under the background of big data era. The methods for analysis, processing and transmission of information data have been improved. With the increase of information volume, there are higher requirements on big data technology for the accuracy and analysis speed of information transmission. With the development of modern computer wireless network communication technology, the transmission control service system will be transformed into a system with expanded functions, which can be directly connected to the data transmission platform, and deal with various problems effectively in information transmission. Besides, a high-quality wireless communication environment needs to be created to promote the development of China's 
wireless network communication technology.

\section{References}

1. Wu Y. Analysis and study discussion on transmission control technology in computer wireless network communication. Wuxian Hulian Keji 2019; 16(10): 1-2.

2. He $X$, Sun J. Brief introduction of transmission control technology in computer wireless network communication (in Chinese). Regional Governance 2019; (9): 185-185.
3. Dai J. Discussion on transmission control technology in computer wireless network communication (in Chinese). Information Technology and Informatization 2019; (8): 130-132.

4. Zhu W, Liu R, Zhang K. Transmission control technology in computer wireless network communication (in Chinese). Electronic Technology \& Software Engineering 2019; (7): 5.

5. Wu Y. Computer wireless network communication from the perspective of transmission control technology (in Chinese). Huadong Keji 2019; (7): 29. 